\title{
A LOW COST NIR BASED EMBEDDED SYSTEM FOR FRUIT QUALITY ASSESSMENT
}

\author{
Arathy Shaji ${ }^{1}$, Liju Philip ${ }^{2}$ \\ ${ }^{1}$ PG Scholar, Department of Electronics Engineering, College of Engineering Chengannur, Kerala, India \\ ${ }^{2}$ Assistant Professor, Department of Electronics Engineering, College of Engineering Chengannur, Kerala, India
}

\begin{abstract}
India is one of the largest producers of fruits in the world.Banana, pineapple and mango are the major fruits produced in our country. In fruits, internal defects, presence of worms, etc. needs to be detected. Recently Computer Vision System (CVS) and image processing are used for evaluating external visual quality factors such as colour, shape, etc. Various non-destructive technologies such as VIS/NIR spectroscopy, ultrasonic, X-ray and MRI are used for evaluating internal quality factors such as sugar content, acid content, etc. But in India, there is lack of such improved technologies and instrumentation for getting right information about quality since these techniques are highly expensive. Thus sorting of fruits is done manually. In manual sorting, it is hardly possible to detect the internal defects.This has resulted in rejection of fruits for export from India. Thus there is a need to develop an embedded system to detect the internal defects including the presence of worms in fruits. In this work NIR based imaging is used for low cost detection of presence of worms in fruits.
\end{abstract}

Keywords: fruits, CVS,NIR, low cost, non-destructive, internal defects, quality

\section{INTRODUCTION}

In India, there is demand for high quality fruits from both local and foreign market. Post-harvest sorting of fruits is done manually. Manual sorting is based on traditional visual quality inspection performed by human operators, which is tedious, time-consuming, slow and non-consistent. It has become increasingly difficult to hire personnel who are adequately trained and willing to undertake the tedious task of inspection. Moreover internal defects are really difficult to sort by the packinghouse workers as those defects are hard or impossible to see. Those unseen defects can weaken peel structure and later lead to visible damage. Thus a faster, reliable and economic internal and external quality evaluation technique is desirable for fruits [2].

Recently image processing is used for evaluating external visual quality factors and VIS/NIR spectroscopy, ultrasonic, $\mathrm{X}$-ray and MRI is used for evaluating internal quality factors such as sugar content, acid content, etc. But these methods are very expensive. Thus there is a need to develop a low cost method to determine the internal quality factors.

NIR methods have been widely used to detect the quality of fruits and vegetables since the NIR optical components (source and detector) are affordable. NIR spectroscopy deals with irradiating the product with NIR light $(750 \mathrm{~nm}$ to 2500 $\mathrm{nm}$ ) and with collecting and analysing the spectrum using spectrum analyser. There are two types of NIR methodsreflectance and transmittance measurement methods. Sweetness grading system is based on NIR reflectance method and can be used for fruits with thin peel such as apple, peer, peach, etc. Transmittance method measures transmitted light through tissues and thus it gives information about overall quality of fruits. It can be used to measure sugar content, acidity, internal defects, etc. [1].

In 1999, Jeroen et al. [6] investigated the transmission and reflectance properties of NIR in apple. They established a relationship between reflectance spectra (obtained by spectrophotometer) and soluble solids content of apple and also between the penetration depth and wavelength. In 2008, Stephen et al.used NIR spectroscopy to predict soluble solids content in mangoes[5]. In 2012 Ayman H. Amer Eissa[7]stated a NIR based orange grading system. The near infrared inspection systems consist of halogen lamps and a spectrophotometer to analyse absorption bands of transmitted light from fruits.

All these methods involve the use of spectrum analyzer to analyze the spectrum. The main objective of this work is to develop a low cost embedded system to detect the presence of worms in fruits using infrared based image processing.

\section{PROPOSED SYSTEM}

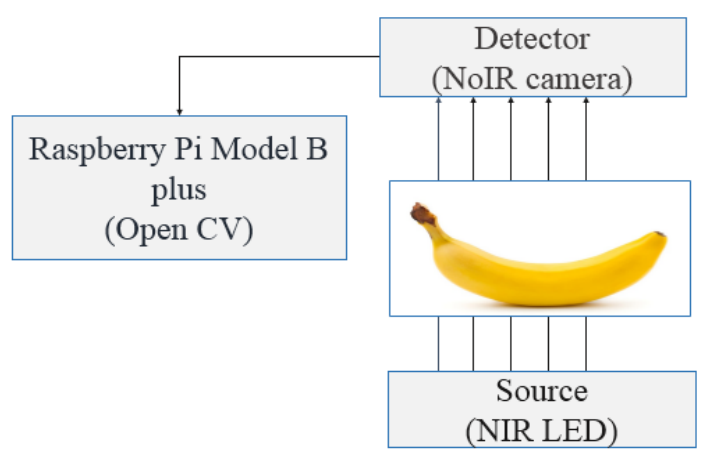

Fig-1:Schematic 
Both ripe and unripe bananas are used for evaluating the NIR imaging-based machine vision system.In the proposed system, the transmitted IR light is used to obtain image of the fruit. The image is captured using Raspberry Pi NoIR camera and then pre-processed. The schematic of the proposed system is shown in Fig-1.

The system primarily consists of three parts:

1. A NIR LED source.

2. A Raspberry Pi NoIR camera (without Infrared cut off filter) to capture the Infrared image.

3. A Raspberry Pi Model B plus-Single Board Computer.

The software module used for image processing is Open Source Computer Vision.

\subsection{Near Infrared LED source}

The first step was to obtain the IR absorbance spectrum of various parts of banana using the spectrophotometerin order to determine the appropriate wavelength of the IR LED source that could be used to detect the presence of worms in banana.

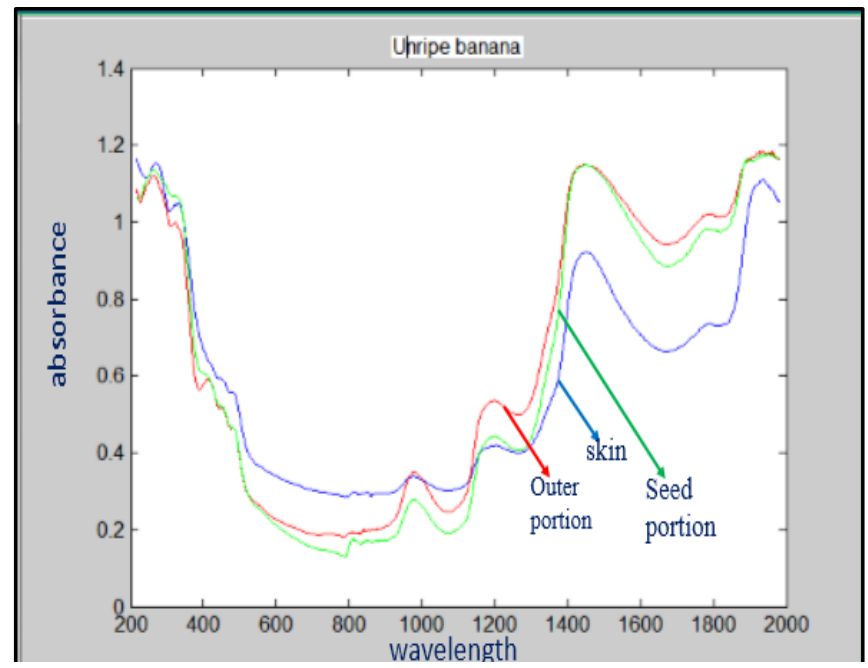

Fig-2: IR spectrum of unripe banana

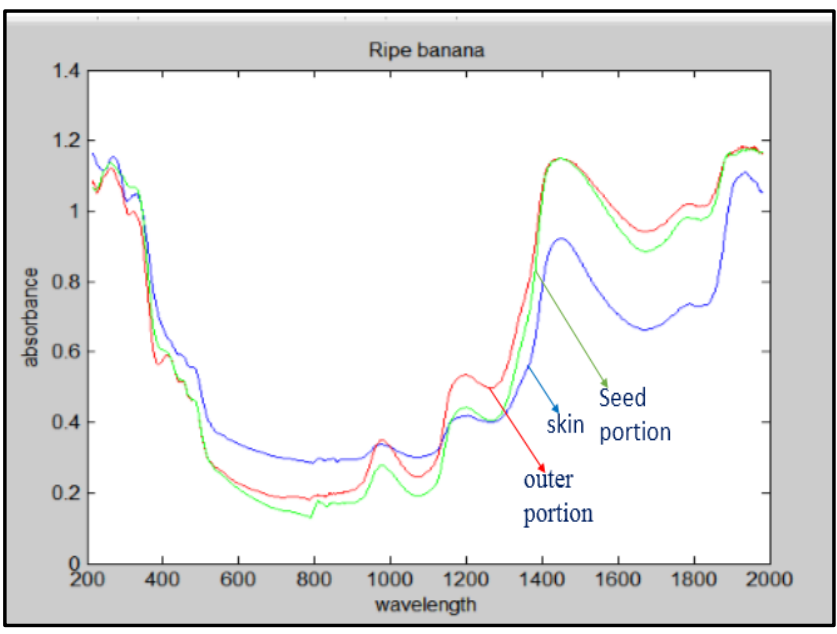

Fig-3: IR spectrum of ripe banana
From the analysis of the above absorbance spectrums of different parts of banana, it is clear that the IR spectrum has minimum absorbance value at approximately $978 \mathrm{~nm}$. Thus in the proposed system $940 \mathrm{~nm}$ IR LED is used to create the sourceconsidering the cost and availability.

The $940 \mathrm{~nm}$ IR LED is inserted into the middle of a foam board painted black and acts as the source as shown in fig-4. The banana is kept above the source and the transmitted light through the incident portion is used to obtain the image from the top. The intensity of the light source is varied using a potentiometer to get a high a contrast image.

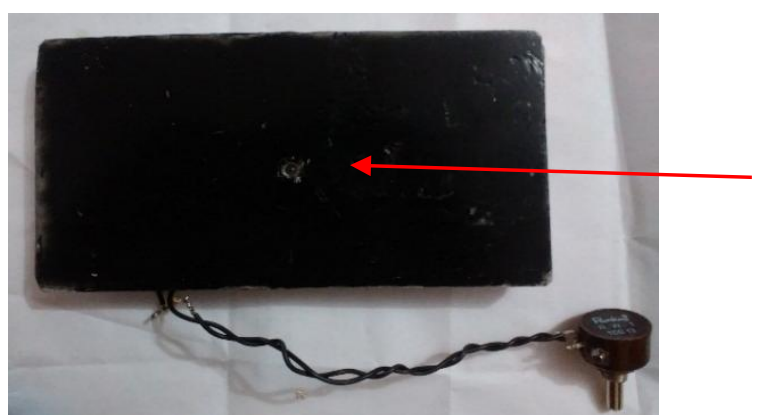

IR LED

Fig-4: Setup of IR LED source

\subsection{Image Acquisition Setup}

Then using foam board, an enclosure is made. The inner walls of this enclosure is coated with black paint (MATT finish) to avoid unwanted reflections. The IR LED source is kept at the bottom of this enclosure. The Raspberry Pi NoIR camera captures images through a hole on the ceiling of the enclosure exactly opposite to the source. The setup is shown in Fig-5.

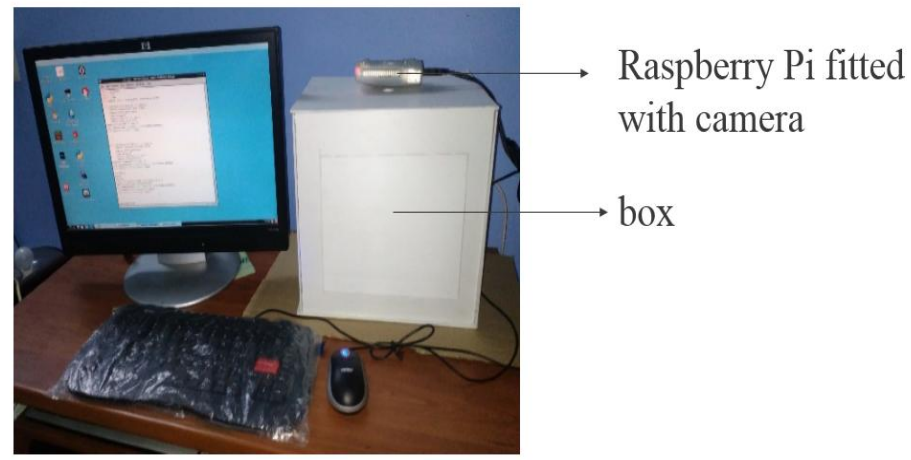

Fig-5: Imaging Setup

The IR light illuminates a portion of the banana and the image is developed in a computer vision unit constructed using Raspberry Pi Model B plus and pre-processed. 


\subsection{Image Acquisition and Pre-Processing}

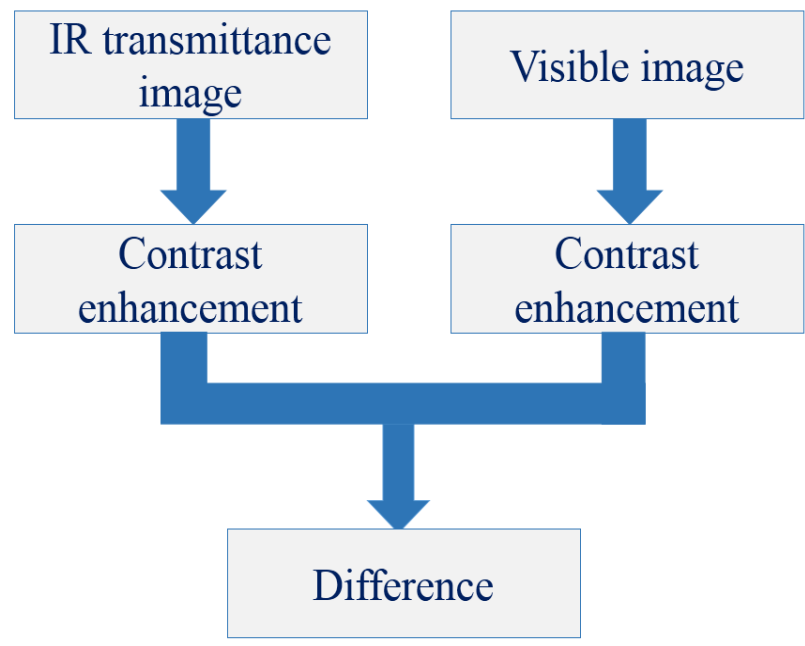

Fig-6: Block diagram

The first step is to obtain the NIR based transmittance and visible reflectance image of a piece of banana with worms inside it. The intensity of the sources are varied for best results.

In the transmittance image, the portion with the worms appear slightly darker. To enhance the contrast of the image, the user is allowed to stretch it beyond its assigned values according to his/her need. With this contrast enhancement technique, slightly darker portion appear darker, hence allowing for easier detection of the presence of worms.

\section{RESULTS AND DISCUSSION}

The histogram of a slice of banana and a worm is taken separately. Histogram is a graphical representation of the tonal distribution in a digital image. It plots the number of pixels for each tonal value. By looking at the histogram of a specific image one will be able to judge the entire tonal distribution at a glance.

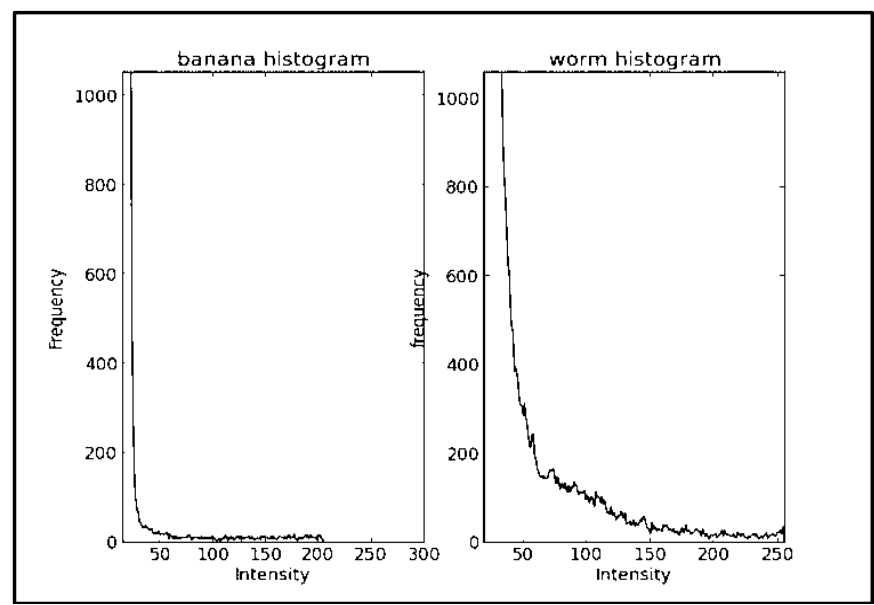

Fig-7:Histogram of banana and worm

From the above histogram of banana slice andworm, it is clear that both of them have different tonal distribution.
A hole of $0.5 \mathrm{~mm}$ diameter and $1.8 \mathrm{~cm}$ depth was made at the centre of a banana slice of $1.8 \mathrm{~cm}$ thickness. Then about 30 worms obtained from mango are inserted into that hole and both transmittance and reflectance image is taken. Now the difference between the IR reflectance and transmittance images is obtained.

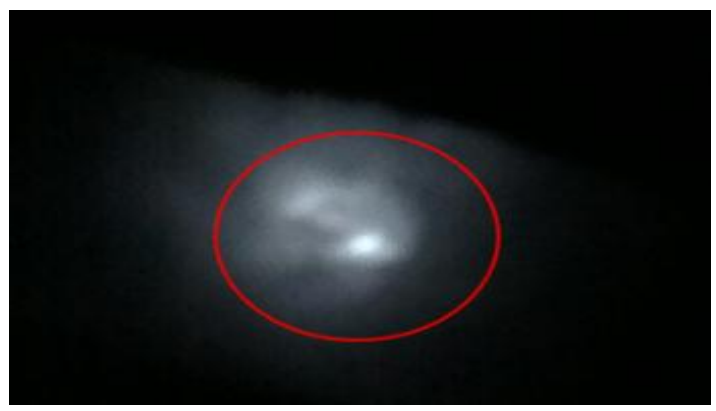

Fig-8: Transmittance image

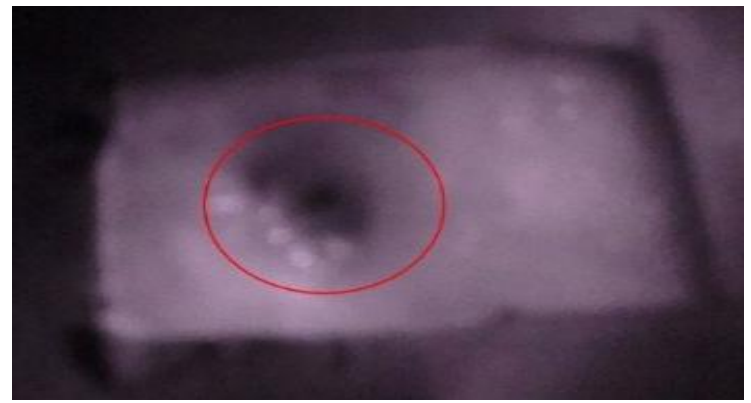

Fig-9: Reflectance image

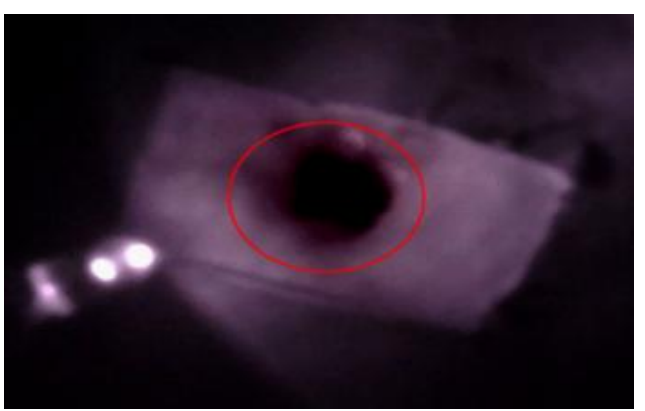

Fig-10: Difference image

Then about 4-5 worms are inserted in $0.7 \mathrm{~cm}$ thick sliceat a depth of $0.2 \mathrm{~cm}$ and image is taken. Now the contrast of the image is enhanced and its difference with the visible image is taken as shown in Fig-12.

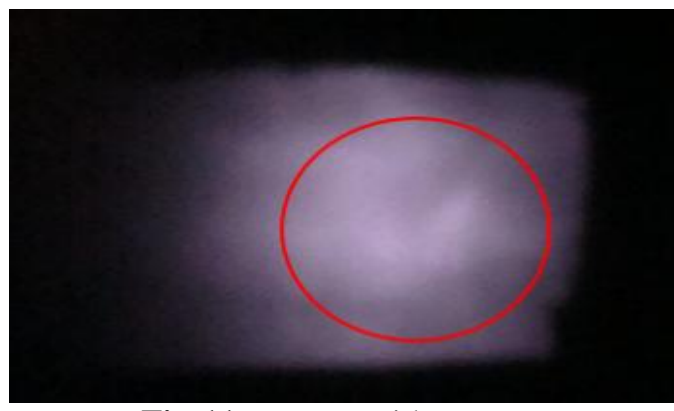

Fig-11: Banana with Worms 


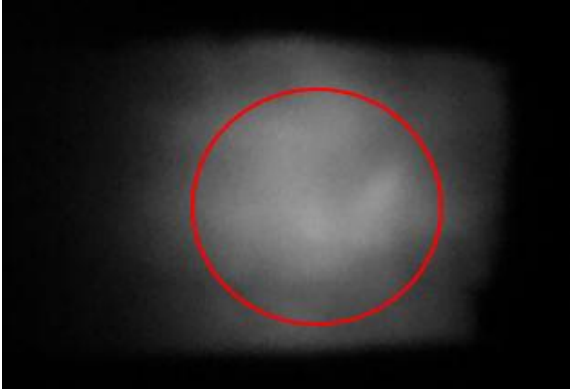

Fig-12: Contrast enhanced

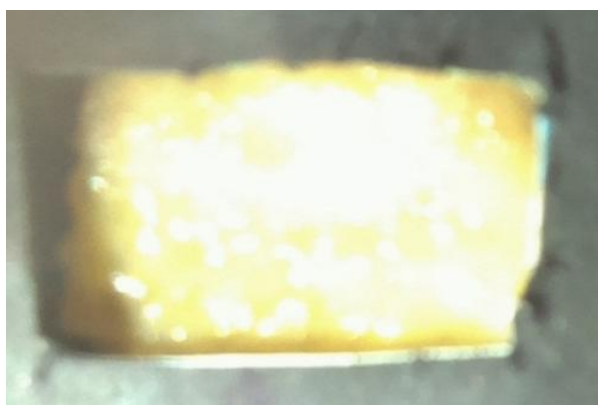

Fig-13:Visible image

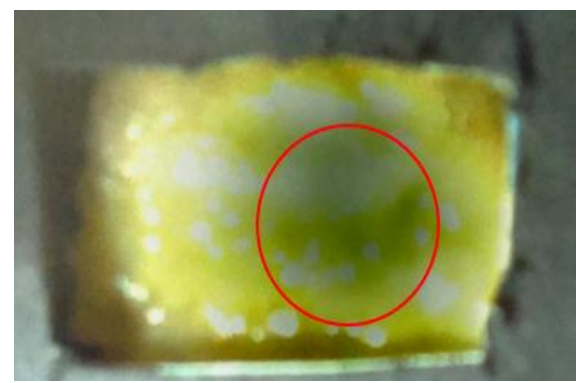

Fig-14: Difference image

From the images it is clear that in IR transmittance image, the region at which worms are present appears brighter and thus in the difference image, the brighter portion appears darker.

\section{CONCLUSION}

NIR imaging can be used as a low cost fruit quality assessment method. The wavelength of LED to be used is determined with the help of spectrophotometer and the spectrum of different parts of banana showed that the absorbance is lowest at approximately $970 \mathrm{~nm}$. Considering the availability and cost, $940 \mathrm{~nm}$ LED is used. Here a single LED of half a watt itself could penetrate through half the banana that is approximately a depth of $2 \mathrm{~cm}$ in case of transmittance. In case of reflectance the penetration depth is less compared to transmittance. The worms have less absorbance and the region in which worms are present in banana appear brighter.

\section{FUTURE SCOPE}

The penetration depth could be increased by using a more powerful IR source having higher wattage. This system couldbe modified to be used with other fruits and for the real time detection of worms in fruits by alternately lighting the fruit with IR and visible light of appropriate intensity and further processing of the images. Thus a complete nondestructive fruit quality assessment system useful for the fruit industry could be designed by making suitable modifications to the proposed system.

\section{REFERENCES}

[1]. K Sang Ha et al. "Non-destructive quality evaluation technologies for fruits and Vegetables", Department of Bio systems and Biomaterial engineering, Korea.

[2]. Amin LotfalianDehkordi et al." A statistical study on precision of ultraviolet (UV) image processing in detecting and measuring apple bruises", International Journal of Bioscience Vol. 4, No. 5, p. 226-231, 2014.

[3]. Nagle et al." Non-destructive mango quality assessment using image processing: inexpensiveinnovation for the food handling industry",Conference on International research on food security, September 19, 2012

[4]. D C Slaughter "Non-destructive maturity assessment methods for mango", Biological and agricultural engineering, Jan 2009.

[5].Yvette Mattley, "Near-Infrared Diffuse Reflection Analysis of Fruit", Ocean Optics.

[6]. Stephen R. Delwiche et al." Soluble Solids and Simple SugarsMeasurement in Intact Mango UsingNear Infrared Spectroscopy", HortechnologyJuly-September 2008 18(3), 2008

[7]. Jeroen et al." Light penetration properties of NIR radiation in fruit withrespect to non-destructive quality assessment", Elsevier Postharvest Biology and Technology 18 (2000) 121-132, 11 October 1999

[8]. Ayman H. Amer Eissa et al." Understanding Color Image Processing by Machine Vision for Biological Materials", INTECH 2012

[9]. G.A. de Oliveira et al. Comparison of NIRS approach for prediction of internal quality traits in three fruit species",Science Direct, Food Chemistry 143(2014)

[10]. Navdeepsih V.Limbadet.al, "Vein Pattern DetectionSystem Using Cost-effective Modified IR Sensitive Webcam", International Journal for Technological Research in Engineering Vol1, Issue 9, May-2014 [11]. Kurita et al.," A double image acquisition system withvisible and UV LEDs for citrus fruit",Journal of Robotics and Mechatronics21 (4):533-540. 